\title{
Sleep Quality and Anxiety Level among College Students
}

\author{
Sreejesh.K.P ${ }^{1}$, Nezra Joseph ${ }^{2}$, Gopika K Sreenivasan ${ }^{2}$ \\ ${ }^{1}$ Assistant Professor, ${ }^{2} 4$ th Year B.Sc. Nursing Student, \\ Amrita College of Nursing, Amrita Vishwa Vidyapeetham University, Health Sciences Campus, Kochi \\ Corresponding Author: Sreejesh.K.P
}

\begin{abstract}
Background: Sleep is essential for prevention of wasteful use of energy, resupply of energy and helps the brain to convert short term memory to long term memory. Poor sleep quality affect person's physical and psychological health.

Objectives: Objectives of the study are to investigate correlation of sleep quality and anxiety level among college students and to find out the association between demographic variables with sleep quality and anxiety level of college students in Ernakulam.

Methodology: Descriptive survey design was adopted for the study. Convenience sampling technique was used to select 100 samples from amrita school of arts and sciences Ernakulum. The tool used for the study consists of structured self-administered demographic profile of college students; Pittsburgh sleep quality index (PSQI) was used to measure the total PSQI score and its 7 sub component and Becks Anxiety Inventory (BAI) was used to measure the self-report measure anxiety. Data was analysed using descriptive and inferential statistics.
\end{abstract}

Result: Majority of the subjects $67(67 \%)$ had poor sleep quality and most of the subjects $76 \%$ had low anxiety and BAI score was highly significant association but had moderately positive correlation with sleep medication and total PSQI Score. All other subcomponents of PSQI had weak positive correlation with anxiety level. Anxiety level of college student had significant association $(\mathrm{p}=0.012)$ association with using of E gadgets

Conclusion: In conclusion of the study decrease in sleep quality increase the anxiety level of college students.
Key words: college students, sleep quality, anxiety

\section{INTRODUCTION}

Sleep, a complex and dynamic process, is important for a number of brain functions, including how nerve cells (neurons) communicate with each other. In fact, our brain and body stay remarkably active while we sleep. Recent findings suggest that sleep place a housekeeping role that removes toxins in our brain that build up while we are awake. ${ }^{[1]}$ Sleep is undoubtedly one of the most essential requirements for the human body to function properly Studies have shown that sleep deprivation can cause psychiatric disorder and dysfunctions such as decreased work efficiency and learning disability. An average adult needs 7-9 hrs of sleep each night, teenagers $9.5 \mathrm{hrs}$ and infants 16hrs/ day. College students are well known for keeping erratic sleep schedules. Even a small reduction in the amount of sleep, especially when combined with a varying sleep schedule, may cause fatigue. When college life starts the students face lots of challenges like new schedules and environment, social obligation as well as academic stress ${ }^{[2]}$ Anxiety may lead to coronary heart disease in later life. ${ }^{[3]}$

An accumulation of research shows that the relationship between disturbed sleep and anxiety is complex and bidirectional. As this relation is better understood, dedicated intervention both on sleep quality and 
anxiety level could prevent the emerging problem in the affected student population, as well as serving as prevention in the young adults. ${ }^{[4]}$

\section{RESEARCH METHODOLOGY}

The present study used a descriptive survey design to collect the data. The study conducted in amrita school of arts and sciences, Ernakulum district of Kerala and data collection conducted on 22/01/2020. Samples were taken by purposive sampling technique and the sample size was 100 . The subjects included in our study were college students who have the age between 18- 21 yrs and who are able to read and write English. The tool used for the study included structured questionnaire, Pittsburgh Sleep Quality Index (PSQI), and Becks Anxiety Inventory (BAI). Structured questionnaire used to collect the demographic data of the students which includes age, gender, year of study, study stream, coffee intake, smoking, liquor intake, economic status, type of family, and area of residence. Pittsburgh sleep quality Index used to assess sleep Pittsburgh Sleep Quality Index (PSQI) contains 19 individual items and 7 sub-components of this scale is sleep quality, sleep latency, sleep duration, habitual sleep efficiency, sleep disturbance, use of sleep medication and daytime dysfunction. Score ranges from 0-21, and higher score is indicative of poorer sleep quality. score less than 5 indicates good sleep quality and total score equal to or more than 5 where considered as poor sleep quality. ${ }^{[5]}$ Beck Anxiety Inventory (BAI) scale is a self-report measure of anxiety. This includes 21 questions, each answers been scored on a scale value of 0 (not at all) to 3 (severely). Higher total scores indicate more anxiety symptoms. In this study, it is designed for individuals who are of $18 \mathrm{yrs}$ of age to 23 years and takes 5-10 minutes to complete. Several studies have found that the Beck Anxiety Inventory to be accurate measure of anxiety symptoms in children and adult. The standardized cut offs are 0-7: minimal anxiety; 8-15: mild anxiety; 16-25: moderate anxiety and 26-63: severe anxiety $^{[6]}$. Data collected after approval obtained from the research committee of Amrita College Of Nursing. Scientific committee and ethical committee of AIMS The study conducted among college students of Amrita Arts and science college students having the age from 18 yrs. to 21 yrs. Samples collected using convenience sampling technique and sample size was 100. Permission to conduct the study collected from Amrita School of Arts and Sciences, Ernakulam. Informed written consent taken from the participants before commencement of the study. Data analyzed with descriptive and inferential statistics. Percentage and frequency used as descriptive statistics and Spearman correlation and chi square test used as inferential statistics. Ethical permission took from the ethical committee, AIMS before data collection

\section{RESULTS}

Socio demographic factors such as age, gender, year of study, type of family, use of illicit drugs, smoking, time of spending in $\mathrm{E}$ gadgets were studied .The study finding shows that majority of the subjects $(61 \%)$ were the age of 20 years and most of them $(87 \%)$ were females. Of the regarding the year of the study most of the subjects $(82 \%)$ were $2^{\text {nd }}$ year under graduate students. Most of them (84\%) were from nuclear family and about majority $(64 \%)$ of them were living with both parents. Most of student's family (99\%) were moderate income family. Only one person was using illicit drugs and none of them are smokers. Most of them (97\%) were using e-gadgets and majority of them (72\%) spend 2-4 hrs in e-gadgets.

Figure I depicts the distribution of subjects based on sleep quality. The finding revealed that majority of the subjects $(67 \%)$ had poor sleep quality. Seven components of total PSQI score also covered. In the subjective sleep quality nearly half of them $(57 \%)$ had fairly good sleep quality and39 (39\%) had $\leq 15$ mins sleep latency About 34 
(34\%) had 16-30mins of sleep latency. Regarding sleep duration 39(39\%) had sleep duration of 5-6hrs and about $37(37 \%)$ had 6-7 hrs. of sleep. Majority of them 73 (73\%) had $>85 \%$ sleep efficiency. Majority of them $69(69 \%)$ had sleep disturbances of less than once a week. Most 92(92\%) of them had use of sleep medication not during past month. About half of them 50(50\%) had a day time dysfunction of once or twice. The anxiety level of the subjects also assessed.

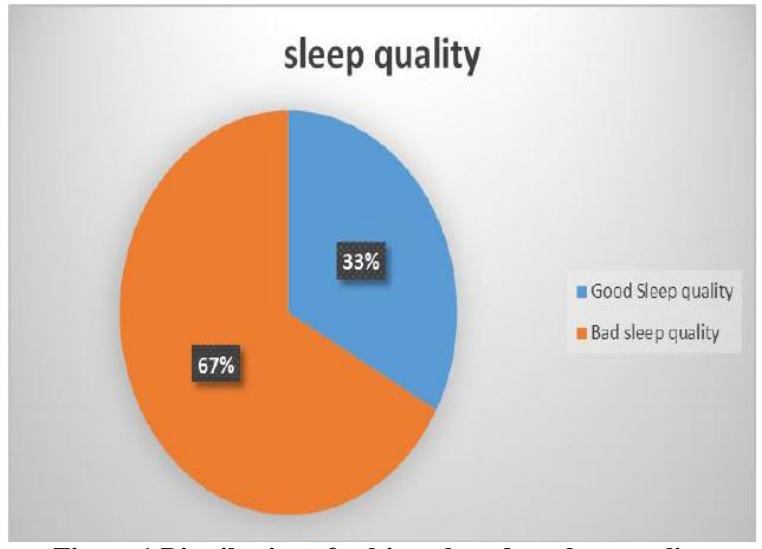

Figure 1.Distribution of subjects based on sleep quality

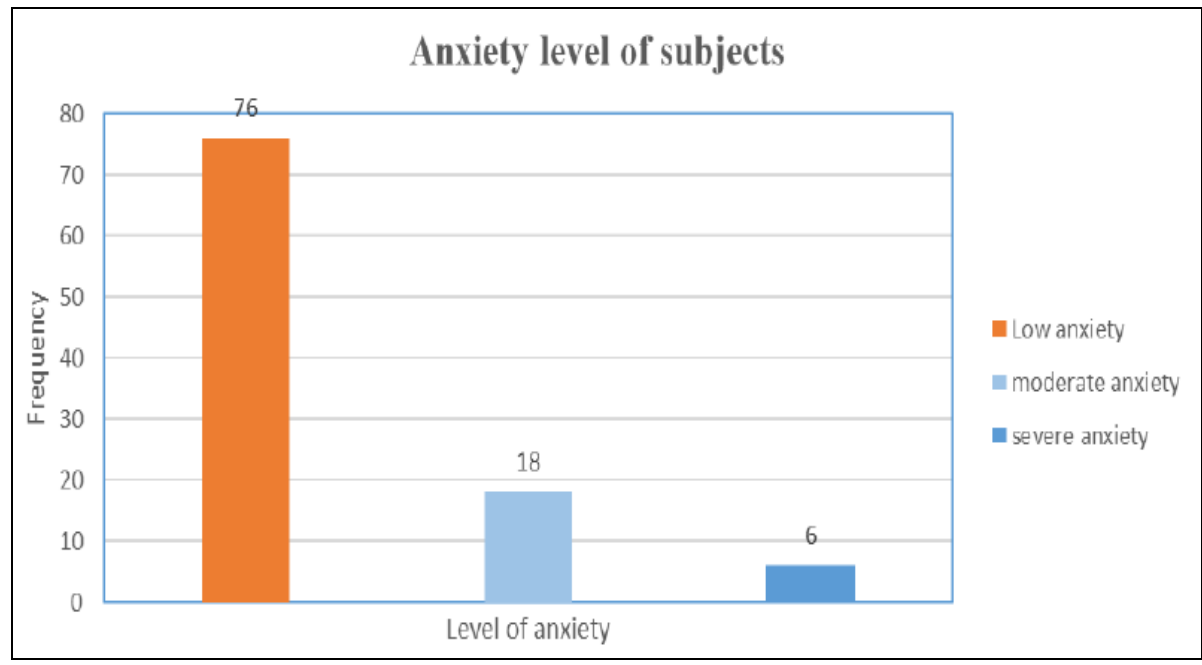

Figure 2: Distribution of subject based on anxiety level

Figure 2 shows that most of the subjects (76\%) had low anxiety level

Table 1: Correlation between sleep quality (Total PSQI Score and anxiety (BAI Score)

\begin{tabular}{|c|c|}
\hline PSQI total & BAI total \\
\hline $\mathrm{R}$ & 0.431 \\
\hline $\mathrm{P}$ & $0.00^{* *}$ \\
\hline ** Correlation is significant at 0.01 level $(\mathrm{P})$
\end{tabular}

** Correlation is significant at 0.01 level $(\mathrm{P})$.
Table I shows that there was positive moderate correlation $(\mathrm{r}=0.431)$ between total PSQI Score and BAI total but the association was highly significant



Figure 3: Scatter diagram showing relationship between anxiety and Total PSQI score 
Figure 3 depicts that there was moderate positive correlation present between sleep quality and anxiety level. In our study there was positive correlation between Total PQSI and its subcomponents with anxiety level of college students. Highly significant $(\mathrm{p} \leq 0.001)$ association found between BAI score with sleep duration sleep medication, day time dysfunction and total PSQI score Among the highly significant association ,moderate positive correlation found present between BAI Score with sleep medication $(r=.410)$ All other subcomponents had weak positive correlation with BAI score. The correlation between the other subcomponents and BAI score was, subjective sleep quality $(\mathrm{r}=0.229)$, sleep latency $(\mathrm{r}=.301)$ Sleep duration $(\mathrm{r}=0.034000$, sleep efficiency $(\mathrm{r}=.026)$, sleep disturbances $(\mathrm{r}=.0 .330)$ and day time dysfunction $(\mathrm{r}=.336)$. There was no significant association found between BAI Score with sleep duration $(\mathrm{P}=0.737)$ and sleep

Table 2: Association between selected demographic variables with sleep quality and anxiety $\mathbf{n}=100$

\begin{tabular}{|c|c|c|c|c|}
\hline \multirow{2}{*}{$\begin{array}{l}\text { Demographic } \\
\text { variables }\end{array}$} & Category & \multirow[t]{2}{*}{ df } & \multirow{2}{*}{$\begin{array}{c}\text { chi square } \\
\text { value }\end{array}$} & \multirow{2}{*}{$\begin{array}{c}p \\
\text { value }\end{array}$} \\
\hline & \begin{tabular}{l|l} 
Yes & No \\
\end{tabular} & & & \\
\hline Usage of E gadgets & 973 & 1 & 6.279 & $0.012 *$ \\
\hline
\end{tabular}

There was significant association $(\mathrm{p}=.012)$ was found between using of $\mathrm{E}$ Gadgets and sleep quality and no significant association found between other demographic variables with sleep quality and anxiety level of college students.

\section{DISCUSSION}

Our study find out that majority of the subjects $(67 \%)$ had poor sleep quality. Similarly community cross sectional study was conducted on prevalence and associated factors of sleep among adults and the setting of the study was Jimma Town, South West Ethiopia. Sample collected through systematic random sampling technique and sample size was 422 adults. The tools used were self administered structured questionnaire to assess sociodemographic variables and Pittsburgh Sleep Quality Index (PSQI) used to assess the sleep quality. The study report supported our study. The majority $(65.4 \%)$ of adults have poor sleep quality ${ }^{[7]}$. Another a descriptive study conducted regarding mobile phone dependence and sleep quality among undergraduate students of Maharajas College Ernakulam $26.4 \%$ of the subjects had mobile phone dependence and among them and Students (23.7\%) who used mobile phone dependence had poor sleep quality. The correlation $(\mathrm{r}=0.347)$ found between mobile phone usage and sleep quality revealed that increased used of mobile phone decreases sleep quality. ${ }^{[8]}$

A correlative study regarding sleep quality and anxiety level among employee conducted in Maramara University. The result finding of our study shows that majority of college students $(67 \%)$ have poor sleep but contradictory to our study results in this study only $38.9 \%$ had poor sleep quality. The Correlation finding between sleep and anxiety in that study found that highly significant association was exist between BAI score and total PSQI score and its seven components except sleep duration and use of sleep medication. Moderate positive correlation present between BAI anxiety score with subjective sleep quality $(\mathrm{r}=0.471)$, sleep disturbance $(\mathrm{r}=0.446)$, day time dysfunction $(\mathrm{p}=0.433)$ and total PSQI score $(p=0.480)$ and all others have weak positive correlation. ${ }^{[9]}$ In our study moderate positive correlation found between total PQSI score and sleep medication use with anxiety level and all others have weakly positive correlation. Similarly a community based cross sectional study conducted about sleep quality and its associated psychosocial factors among elderly in rural population. The setting of study of the Njarrackal Panchayat, Ernakulam. The sample size of the study was 170 and sample selected using simple random sampling. Pittsburgh sleep quality Index (PSQI), General Anxiety Disorder 7 items (GAD-7)scale, geriatric depression score (GDS 5 items) and self administered general socio demographic questionnaire were the tools used for the study. The result 
of the study supporting our study in the case of sleep quality of subjects. Majority of the subjects $(72.4 \%)$ had poor sleep quality .In this study significant association $(\mathrm{p}=0.017)$ was found between general anxiety disorder and GPSQI ${ }^{[10]}$. Similarly a descriptive study conducted to estimate the prevalence and determinants of somatization and anxiety among adult women in urban population, Kochi. The sample size used for the study was 1210 adult women. The study finding showed that $40.8 \%$ of subjects had somatization and $28 \%$ had anxiety disorder. One of the independent determinants of anxiety was sleep problem $(\mathrm{OR}=1.64$, C.I= $1.77-2.9)^{[11] .}$

Another objective of our study to find out the association between demographic variables with sleep quality and anxiety level. Similarly a descriptive cross sectional study done to understand sleep quality and sleep duration of healthy adults and to identify related factors. The study conducted among healthy adults have the age more than 19 years and came to an electroencephalogram centre located in Seol, Korea and data collection period was from March 2015-March 2016 and the sample size was 172 . The tool used for the study was structured questionnaire to assess socio demographic variables, Pittsburgh sleep quality to assess sleep quality, Beck Depression scale to assess depression, fatigue severity scale to assess fatigue. As our study results majority $(59.6 \%)$ of the subjects had poor quality sleep. In this sleep quality had moderate correlation with depression, fatigue and anxiety. Highly significant but moderate positive correlation $(\mathrm{r}=0.31, \mathrm{p}<0.01)$ present between sleep quality and anxiety level. ${ }^{[12]}$

Similarly a cross sectional study regarding sleep quality in college students conducted from Chandigarh. The data collection period from October 2013 to December 2014.In this there was prevalence of poor sleep quality was significantly associated with female sex. However no significant association was observed between stream, year of study and prevalence of poor sleep quality. There was statistically significant difference between prevalence of poor sleep quality between with $\operatorname{sex}(\mathrm{p}=.001)$ with physical activity $(\mathrm{p}=0.009)$, consumption of tea/coffee (0.006) and smoking $\mathrm{p}=010)^{[13]}$.

\section{CONCLUSION}

In conclusion of the study majority of the students had poor sleep quality and there is a moderate positive correlation between sleep quality and anxiety level among the college students. The health awareness programme regarding sleep hygiene is necessary and thus improved sleep reduce the anxiety level.

\section{Acknowledgement: None}

Financial support: self

\section{Conflict of Interest: None}

\section{Source of Funding: None}

Ethical Approval: Ethical permission took from the ethical committee, AIMS before the data collection.

\section{REFERENCES}

1. Basics B. Understanding sleep. National Institute of Neurological Disorders and Stroke, Bethesda. 2006.

2. Sarita, M, Sukhwant B. Study of sleep pattern and sleep problems of under graduate students from different professional courses. Indian Journal of Basic and Applied Medical Research.2016; 5(2):16-28.

3. Dahale AB, Menon JC, Jaisoorya TS. A narrative review of the relationship between coronary heart disease and anxiety. Iran $\mathbf{J}$ Psychiatr Behav Sci 2017;11(3)

4. Dinis J, Bragança M. Quality of sleep and depression in college students: a systematic review. Sleep Science. 2018 Jul; 11(4):290.

5. Smyth CA. Evaluating Sleep Quality in Older Adults: The Pittsburgh Sleep Quality Index can be used to detect sleep disturbances or deficits. AJN The American Journal of Nursing. 2008 May 1;108(5):4250 . 
6. Beck AT, Epstein N, Brown G, Steer RA. An inventory for measuring clinical anxiety: psychometric properties. Journal of consulting and clinical psychology. 1988 Dec;56(6):893.

7. Berhanu H, Mossie A, Tadesse S, Geleta D. Prevalence and associated factors of sleep quality among adults in Jimma Town, Southwest Ethiopia: a community-based cross-sectional study. Sleep disorders. 2018 Apr 22;2018.

8. Anju Philip T, Aswathy Krishnan S, Saju A, Athulya N. Mobile phone dependence and sleep quality among undergraduate students. Indian J Forensic Med Toxicol 2019;13 (3):11-15.

9. Teker AG, Luleci NE. Sleep quality and anxiety level in employees. Northern Clinics of İstanbul. 2018;5

10. George S, Paul G, Paul N. Study on sleep quality and associated psychosocial factors among elderly in a rural population of Kerala, India. International Journal of
Community Medicine and Public Health. 2018 Jan 24; 5(2):526-31

11. Babu AR, Sreedevi A, John A, Krishnapillai V. Prevalence and determinants of somatization and anxiety among adult women in an urban population in Kerala. Indian J Community Med 2019;44(5):S66S69.

12. Shim, Joohee, and Seung Wan Kang. Behavioral factors related to sleep quality and duration in adults. Journal of Lifestyle Medicine 7.1 (2017): 18.

13. Kaur, Gurjeet. A study on sleep quality among college student. JSM Brain Scs. 2018 Aug 31; 3 (1)

How to cite this article: Sreejesh.K.P, Joseph N, Sreenivasan GK. Sleep quality and anxiety level among college students. International Journal of Research and Review. 2021; 8(10): 271-276. DOI: https://doi.org/10.52403/ijrr.20211036 